\title{
Psychometric Properties of the Attitude towards Pressure Ulcer Prevention Instrument (APuP) among Nurses
}

\author{
Mehdi Heidarzadeh ${ }^{1}$, Habibeh Moharramzadeh ${ }^{2 *}$, Nader Moharramzadeh $^{3}$
}

1. Nursing and Midwifery School, Ardabil University of Medical sciences, Ardabil, Iran

2. Alzahra teaching hospital, Tabriz University of Medical Sciences, Tabriz, Iran

3. Medical Emergency and Accident Management Center, Tabriz University of Medical Sciences, Tabriz, Iran

Received: 12 May 2021

Accepted for publication: 7 July 2021

[EPub a head of print-31 July 2021]

Payesh: 2021; 20 (4): 487- 495

\begin{abstract}
Objective (s): Pressure ulcers are a significant health problem worldwide. Positive attitudes towards pressure ulcer prevention have a positive impact on preventive care. This study aimed to determine the psychometric properties and factor structure of the Attitude towards Pressure ulcer Prevention instrument (APuP) in Iranian nurses.

Methods: This was a cross sectional study on a sample of nurses working in ICUs and the internal medicine, surgery, orthopedics, cardiovascular, neurology, and neurosurgery departments in four teaching hospitals in Ardabil, Iran. construct validity (confirmatory factor analysis), internal consistency, and test-retest analyses were performed to determine the psychometric properties of the APuP.

Results: In all 190 nurses partipated in the study. With regard to fitness indices including GFI 'AGFI ،CFI 'RMSEA factor structure of the APuP with five factor and 13 items were confirmed. The Cronbach's alpha for the overall APuP was 0.70 , and correlation coefficient between test-retest was $0.85(\mathrm{ICC}=0.85, \mathrm{p}<0.0001)$.

Conclusion: The findings showed that the Persian version of Attitude towards Pressure ulcer Prevention instrument could be used as a standard tool to assess nurses' attitudes about pressure ulcer prevention.
\end{abstract}

Key Words: Pressure ulcer, psychometric, Attitude, nurse, Prevention

\footnotetext{
${ }^{*}$ Corresponding author: Alzahra teaching hospital, Tabriz University of Medical Sciences, Tabriz

E-mail: h.moharramzadeh13@gmail.com
} 


\section{روانسنجى ابزار نكرش نسبت به يِشَيرى از زخم فشارى (APuP) در برستاران}

مهيدى حيدر زاده'، حبيبه محرم زاده ‘*، نادر محرم زاده

ا. دانشكده يرستارى و مامايى، دانشعاه علوم يزشكى اردبيل، اردبيل، ايران

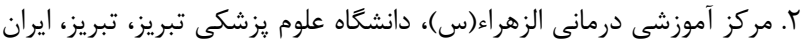

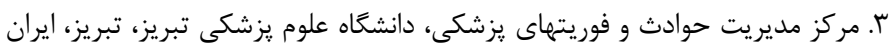

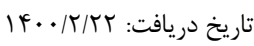

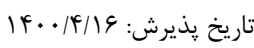

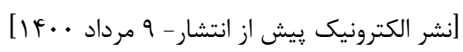

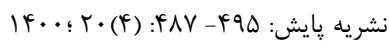

جكيده مقدمه: زخمهاى فشارى يك مشكل بهداشتى مهم در سراسر جهان است. يرستاران به عنوان يكى از اعضاى اصلى تيم سلامت نقش مهمسى در ريشــيرى،

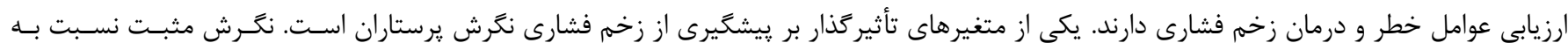

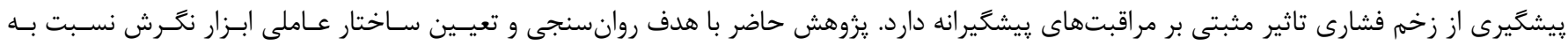

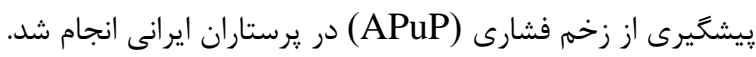

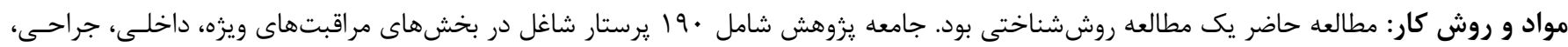

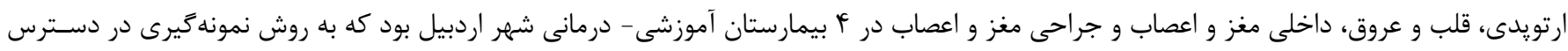

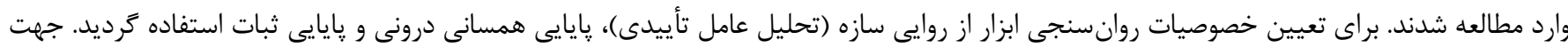

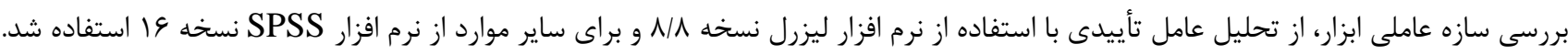

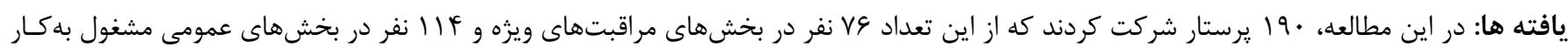

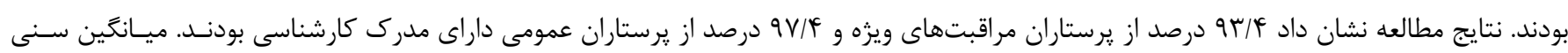

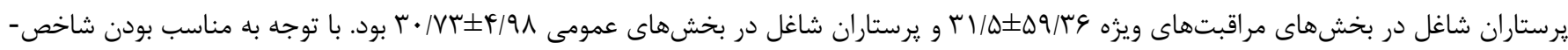

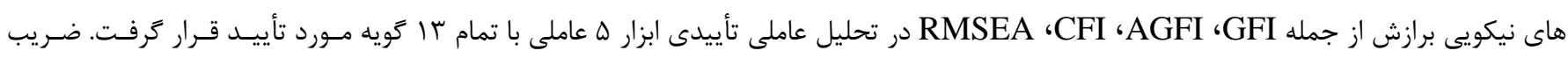

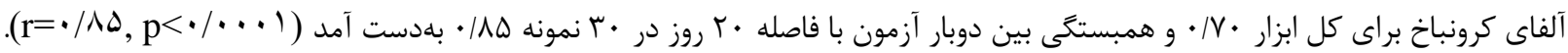

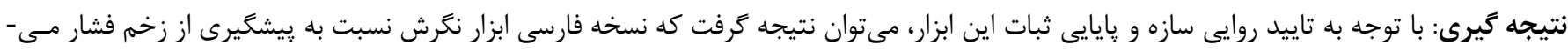

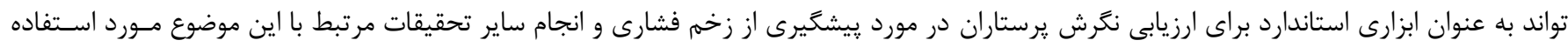
قرار گيرد. كليدوازه: زخم فشارى، روانسنجى، نكرش، برستار، بيشگيرى

كد اخلاق: IR.ARUMS.REC.1398.309 
اين زمينه طر احى شده است ابزار ارزيابى نخرش نسبت به پيشخيرى

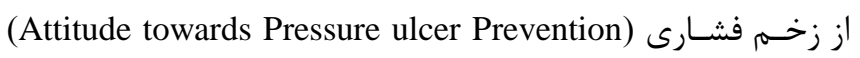

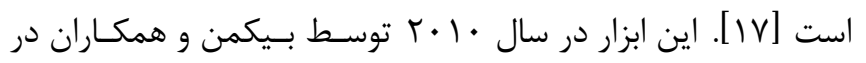

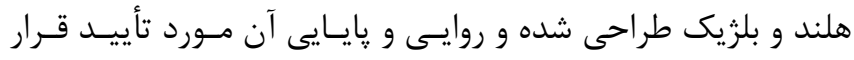

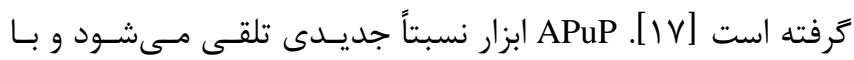

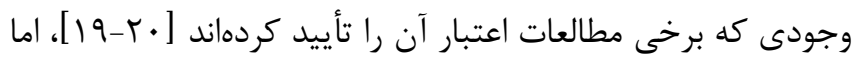

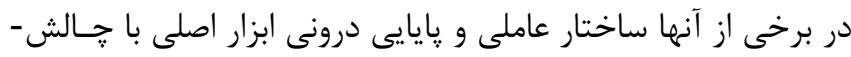

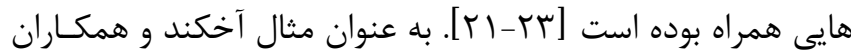
در مطالعه خود با حذف يكى از تويه هاى ابزار نشان دادند كه مــدل

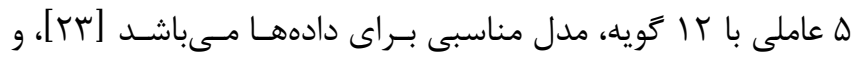

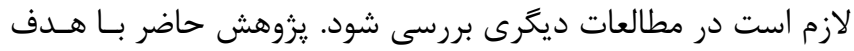

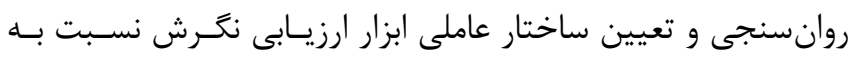

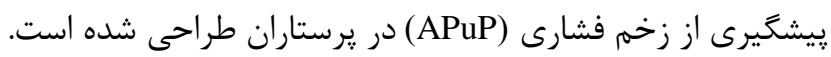

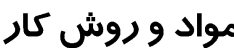

يزوهش حاضر يك مطالعه روششناختى مىباشد كه به روانسنجى

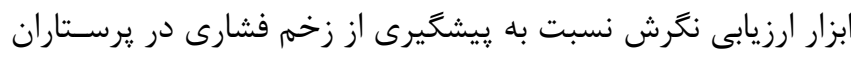

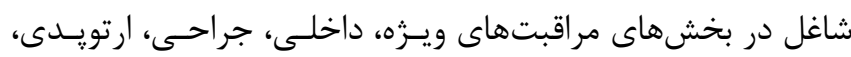

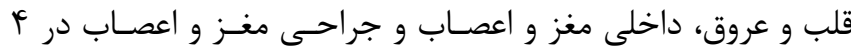
بيمارستان آموزشى - درمانى شهر اردبيل يرداخت. تعداد نمونـهـ لازم

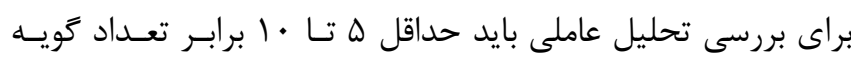

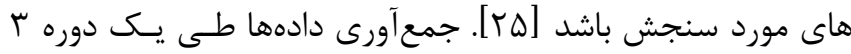

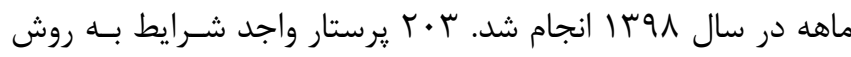

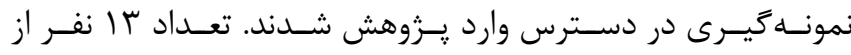

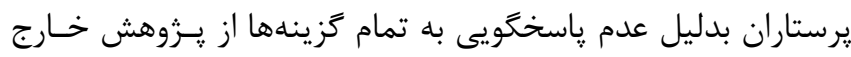

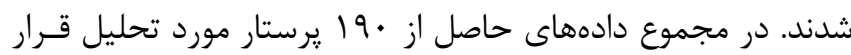

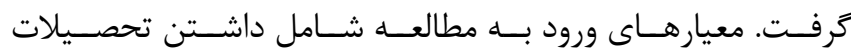

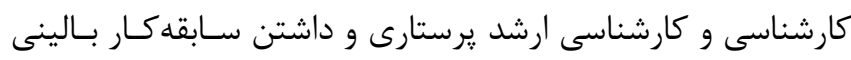
يرستارى حداقل براى يكسال و عدم شركت در برنامههاى آهـوزش

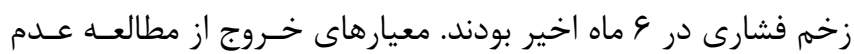

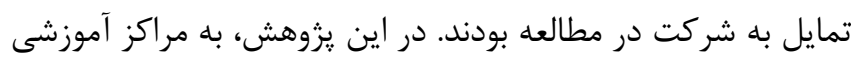

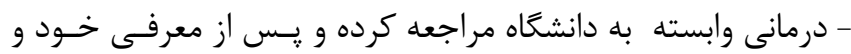

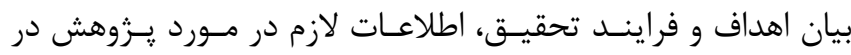

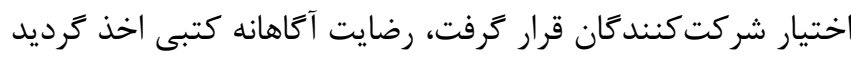

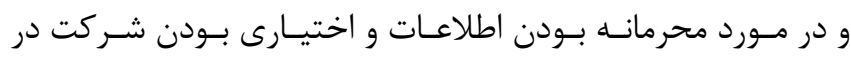
مطالعه به شركت كنندكان توضيح داده شد.
زخم فشارى كه طبق تعريف انجمن ملى مشـاوره زخسم فشـارى بــهـ

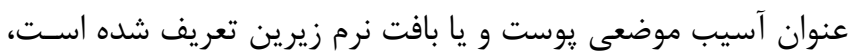

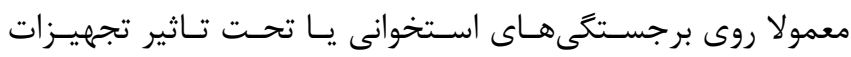

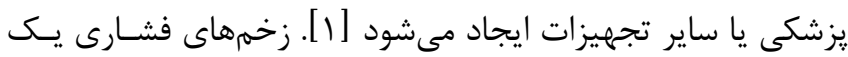

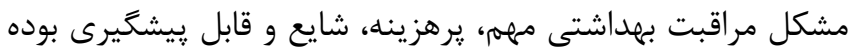

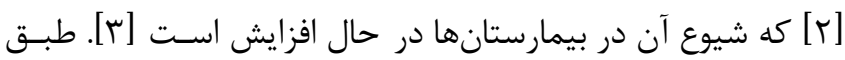

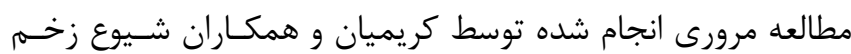

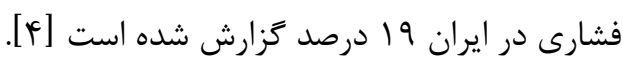

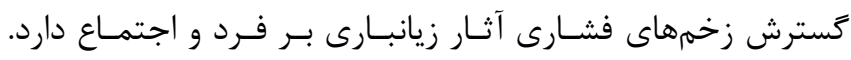

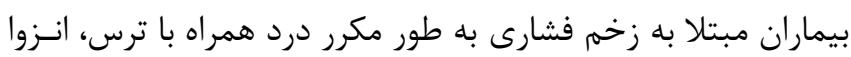

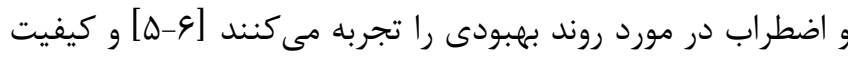

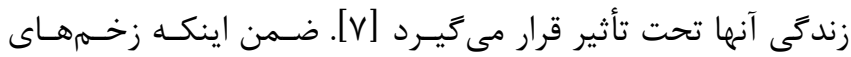

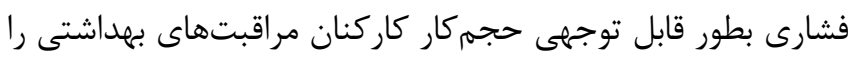

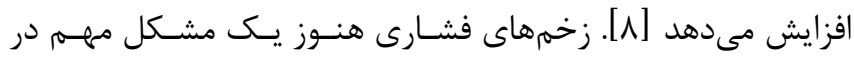

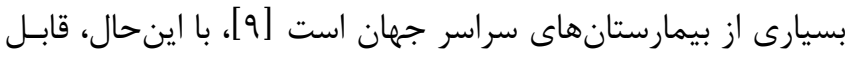

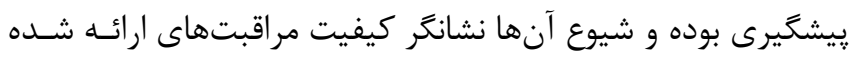

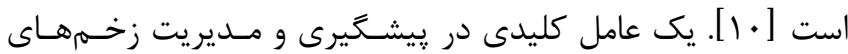

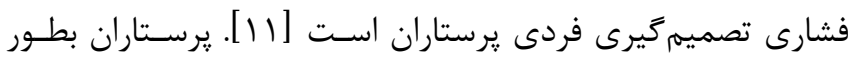

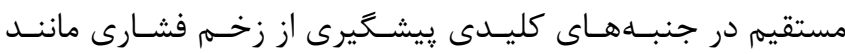

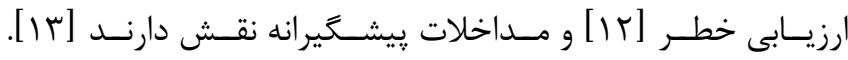

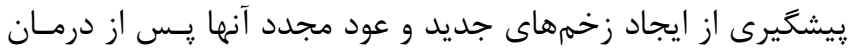

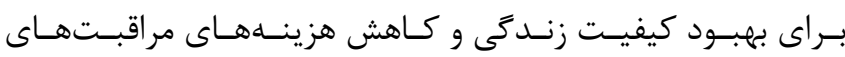

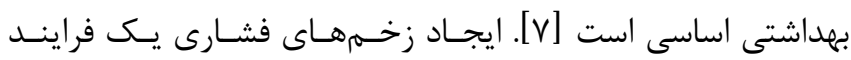

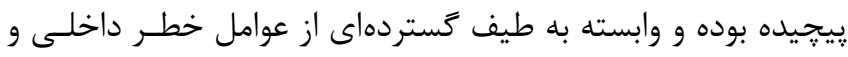

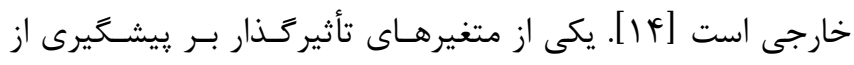

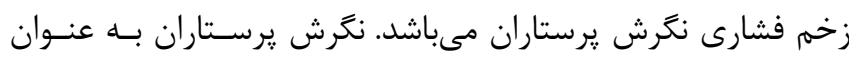

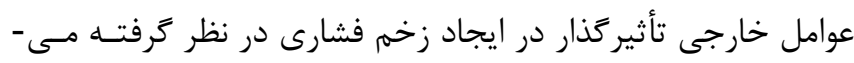

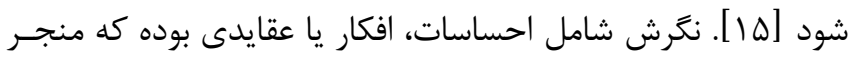

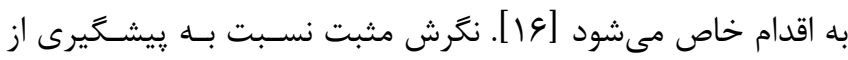

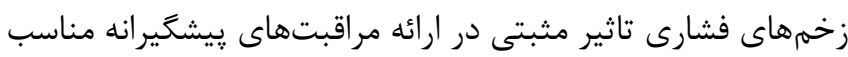

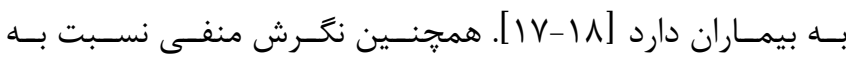

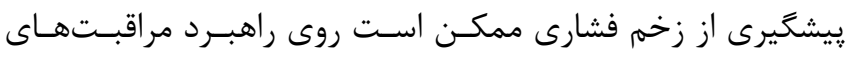

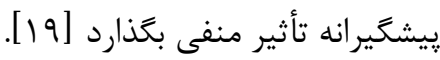

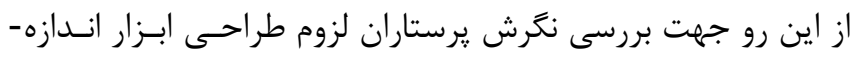

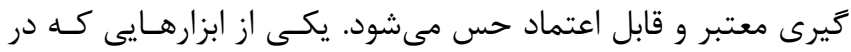


نشريه يثروهشده علوم بهداشتى جهاددانشگاهى

(AGFI)، شـاخص بــرازش تطبيقـى (CFI) و ريشــه متوسـط مربــع خطاى تقريبى (RMSEA) مورد استفاده قرار گرفت. براى بررسى همسـانى درونسى از محاسـبه ضــريب آلفـاى كرونبـاخ

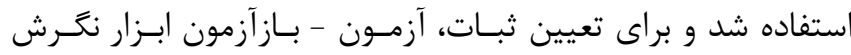
نسبت به ييشخيرى از زخم فشارى مورد بررسى قرار گرفت. به ايـن

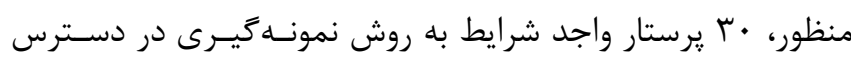

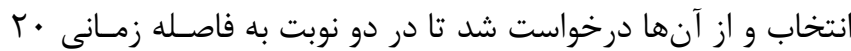

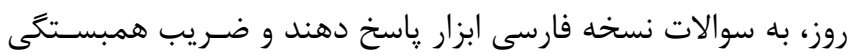

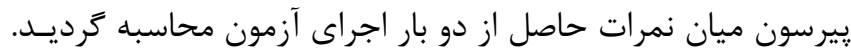

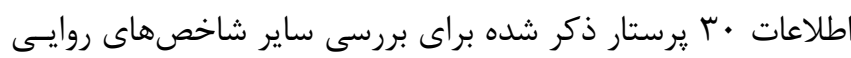

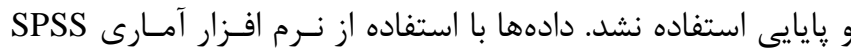

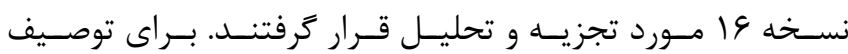

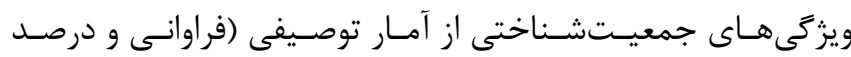

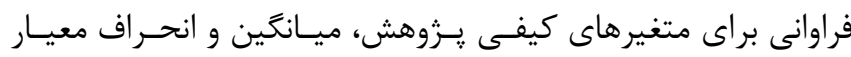

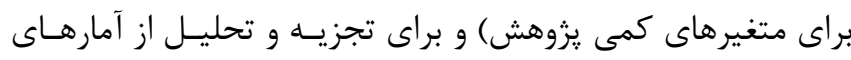
يارامتريك به دليل ماهيت كمى و توزيع نرمال دادهها استفاده شـد.

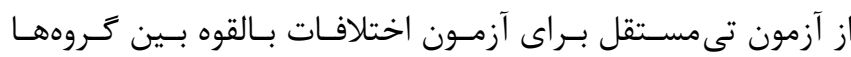

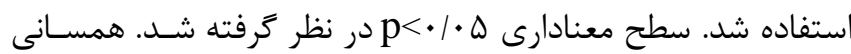

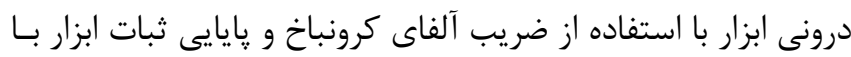

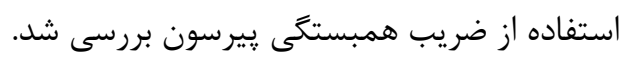

\section{يافتهها}

در مجموعَ دادههاى حاصل از • 19 يرستار مورد تحليل قرار گرفت.

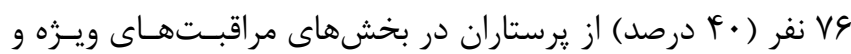

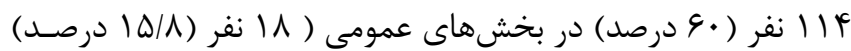

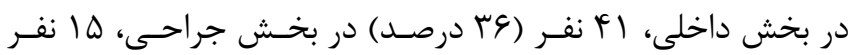

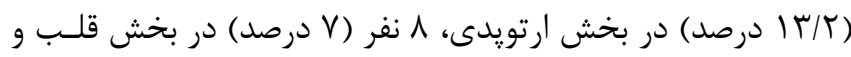

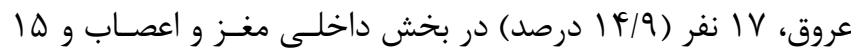
نفر (T/Kا درصد) در بخش جراحى مغز و اعصاب) مشـغول بـهـــار بودند. اكثر شركت كنندكان در بخشهاى مراقبتهـاى ويـرثه (

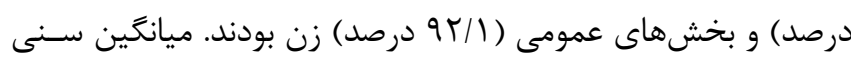

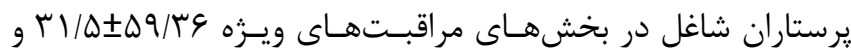

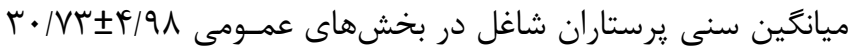

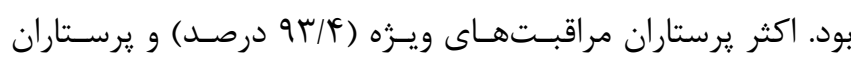

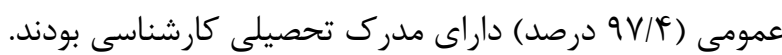

به منظور جمع آورى دادهها از فـرم اطلاعـات جمعيـت شـناختى و

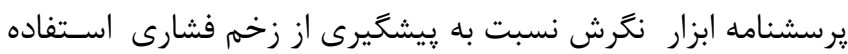

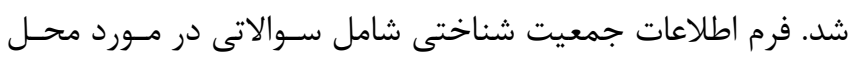
فعاليت، سن، جنسيت، وضعيت تأهل، سطح تحصيلات، بخش محل سل

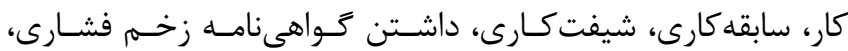

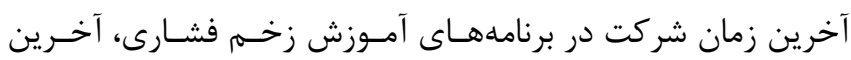

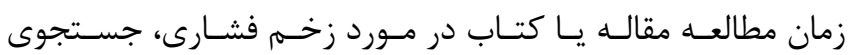

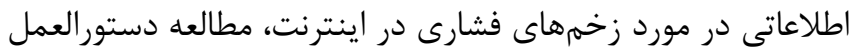

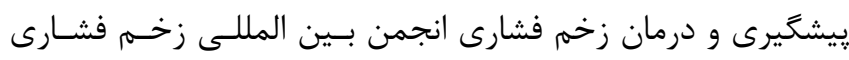
و انجمن مشاوره زخم فشارى ارويا (NPUAP)

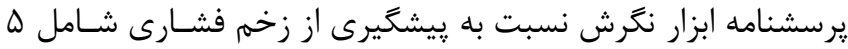

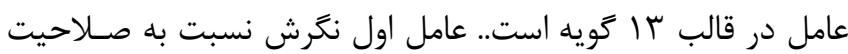

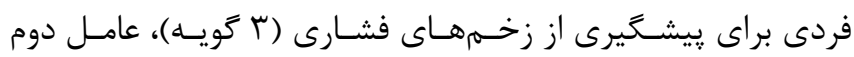

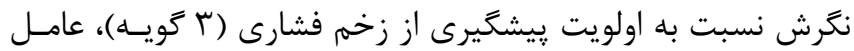
سوم نخرش نسبت به تاثير زخمهاى فشارى (ب گويه)، عامل جهـارم

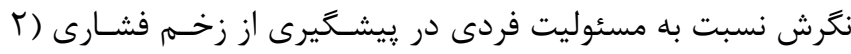

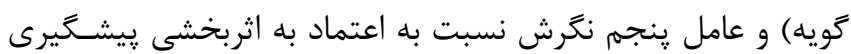

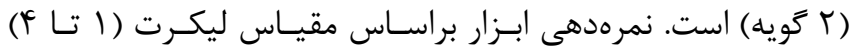

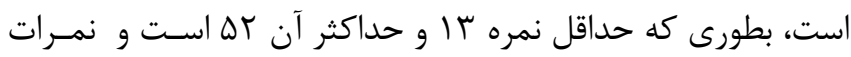
بالاتر نخرش مثبت ترى را نشان مى بـهد [IV] در مطالعه حاضر براى ترجمه مقياس از كامهاى معرفى شده توسط ماى

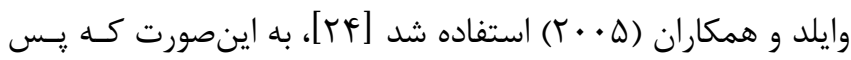
از مكاتبه الكترونيكى با طراح ابـزار و كسـب مجــوز اسـتفاده، ابـزار

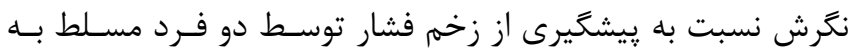

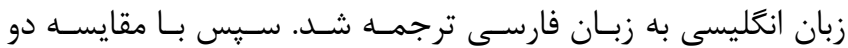

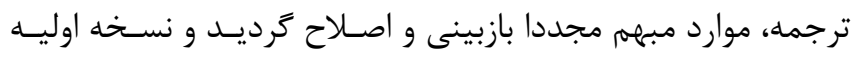

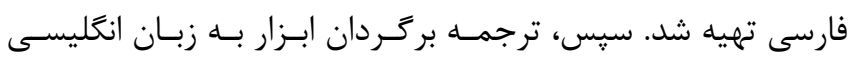
توسط سومين فـرد آشــا بـهـ زبـان انغليسـى انجـام شـده و نسـخه

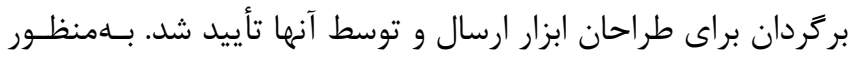

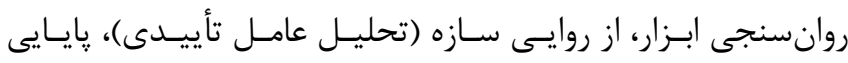

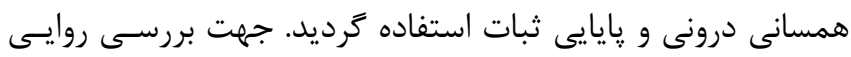

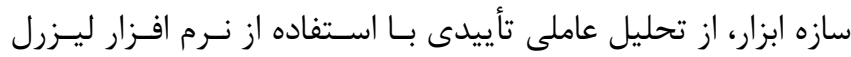

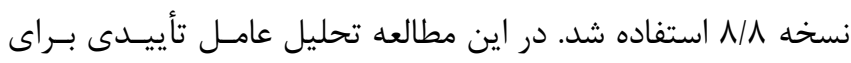

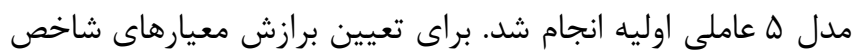

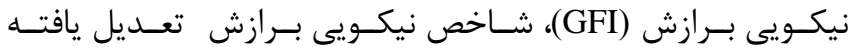


شاخصهـــاى نيكــويى بـــرازش شــامل شـاخص نيكـويى بـرازش

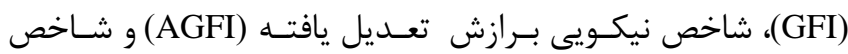

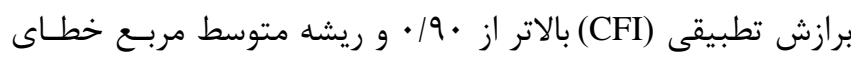

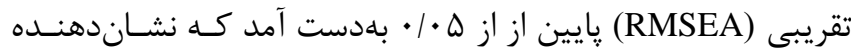

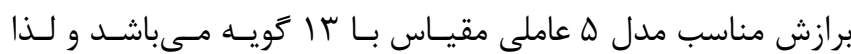

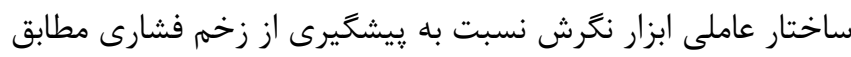

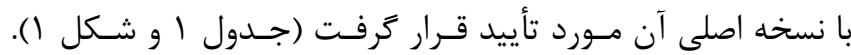
براى تعيين همسانى درونى از ضريب آلفاى كرونباخ استفاده كرديد.

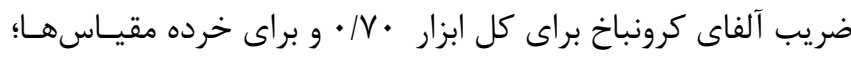

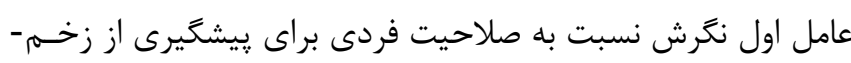

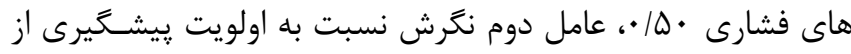

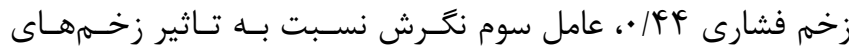

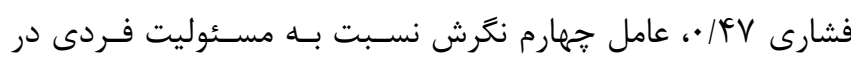

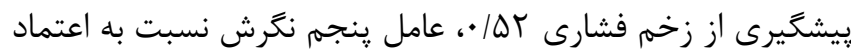

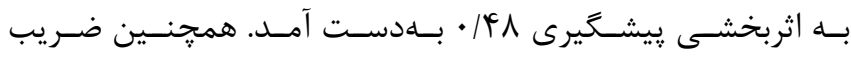

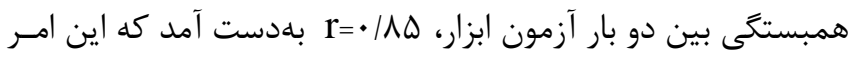

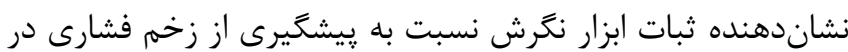

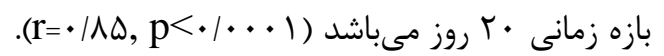

بيشترين فراوانى در رابطه با سابقه كارى در گروه يرستاران مراقبت-

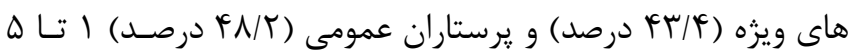

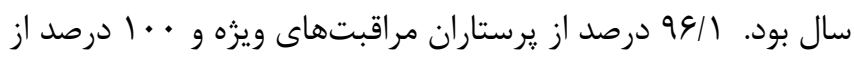
يرستاران عمومى فاقد تواهينامه زخم فشارى بودند.

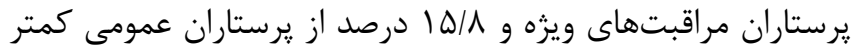

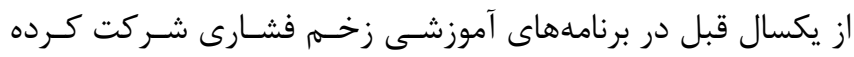

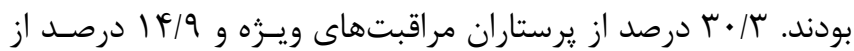

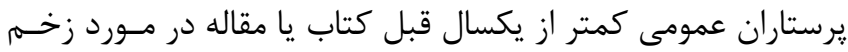

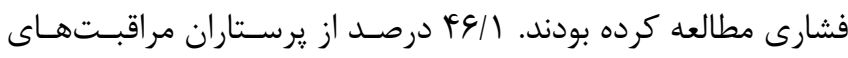

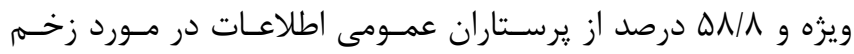

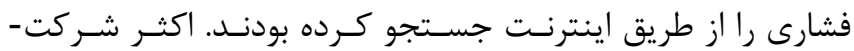

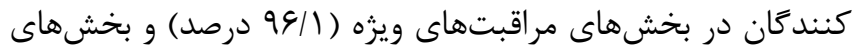

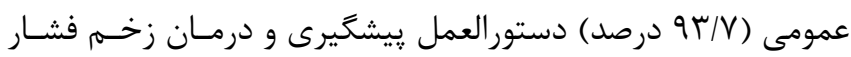

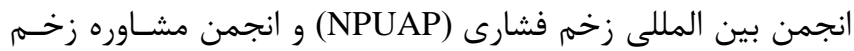

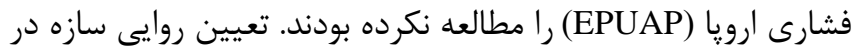

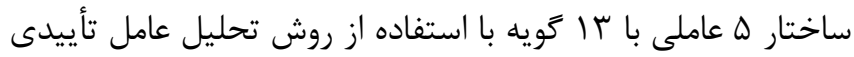

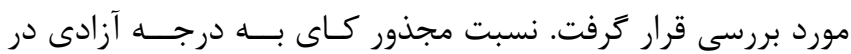

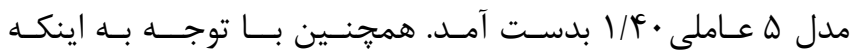

جدول ا: نتايج تحليل عاملى تأييدى APuP

\begin{tabular}{|c|c|c|c|c|c|c|c|c|c|c|c|}
\hline \multirow[b]{2}{*}{ RMSEA } & \multirow[b]{2}{*}{ GFI } & \multirow[b]{2}{*}{ AGFI } & \multirow[b]{2}{*}{ IFI } & \multirow[b]{2}{*}{ CFI } & \multirow[b]{2}{*}{ RFI } & \multirow[b]{2}{*}{ NFI } & \multirow[b]{2}{*}{ NNFI } & \multirow[b]{2}{*}{$\chi^{2} / \mathrm{Df}$} & \multicolumn{3}{|c|}{ شاخصهاى برازش } \\
\hline & & & & & & & & & $\mathrm{df}$ & $\chi^{2}$ & مدل \\
\hline$\cdot 1 \cdot 4 \Delta$ & .194 & .19. & .198 &.$/ 9 \Delta$ & $\cdot / \Lambda$. & 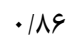 & .94 & $1 / 4$. & $\Delta \Delta$ & $V G / F \mid$ & عامل \\
\hline
\end{tabular}

df: Degree Freedom; $\chi^{2} /$ df: chi-square/degrees of freedom; NNFI: Non- Normed Fit Index; NFI: Normed Fit Index; RFI: Relative Fit Index; CFI: Comparative Fit Index; IFI: Incremental Fit Index; AGFI: Adjusted Goodness of Fit Index; GFI: Goodness of Fit Index; RMSEA: Root Mean Square Error of Approx 


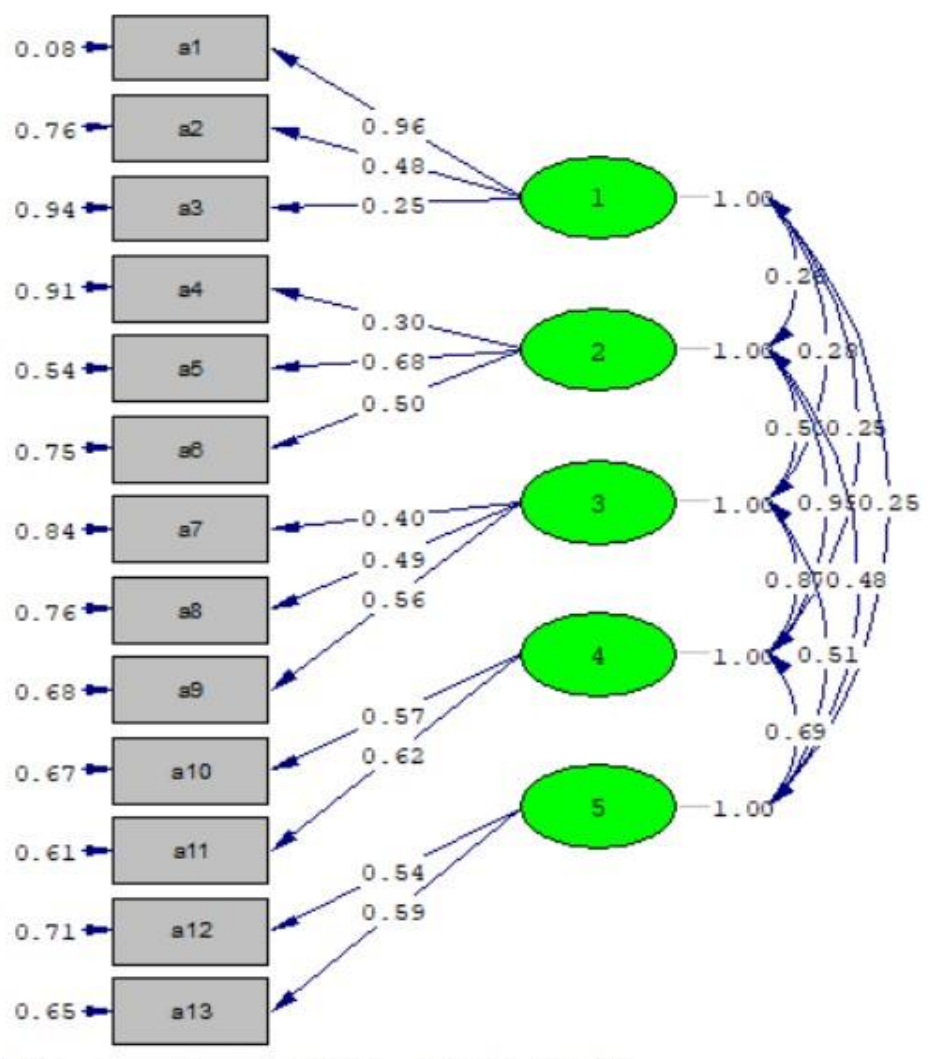

Chi-Square=76.41, df $=55, \mathrm{P}$-value $=0.02967, \mathrm{RMSEA}=0.045$

a1-a13

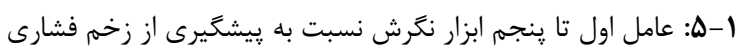

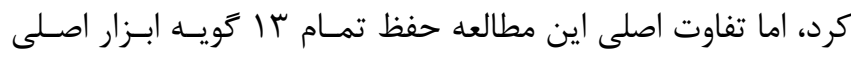

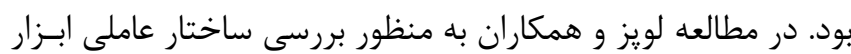

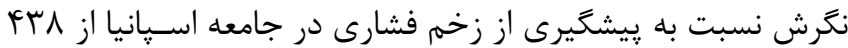

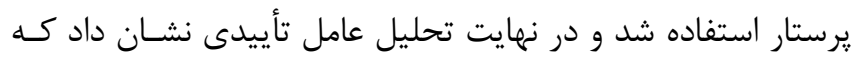

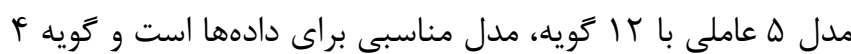

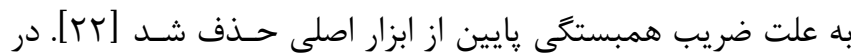
مطالعه آخكند و همكاران نيز به منظور بررسى ساختار عـاملى ابـزار

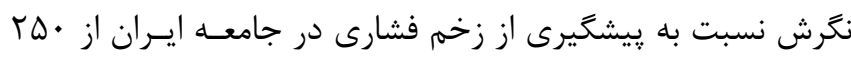

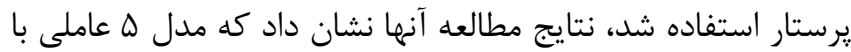

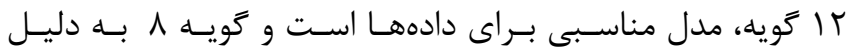
بارگذارى ضعيف عوامل از ابزار اصلى حذف شد [سr]. اما در مطالعه

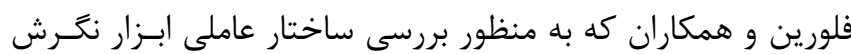

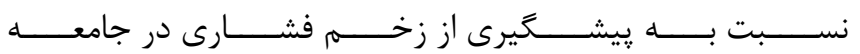

\section{بحث و نتيجه گيرى}

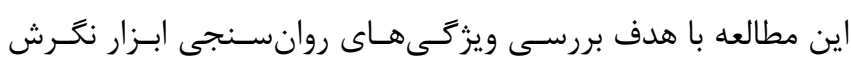

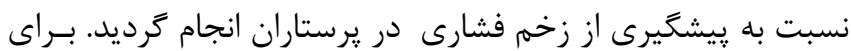

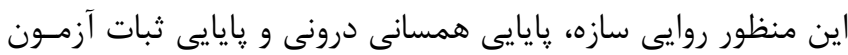

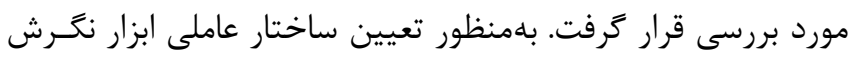
نسبت به يِيشگيرى از زخم فشارى، از تحليل عاملى تأييدى استفاده شد. با توجه به نتايج شاخصهاى نيكويى برازش تمـام سا گويـه در

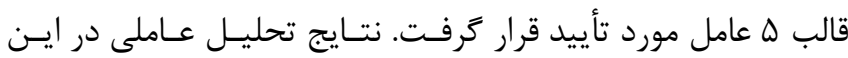

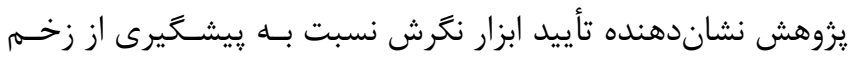

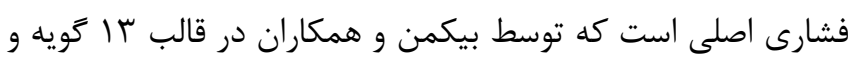

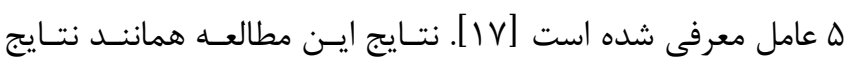

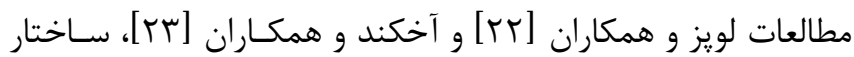

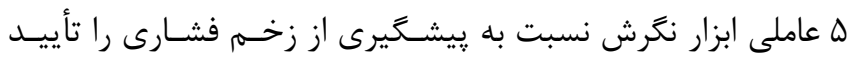


در طول زمان ثبات خود را حفظ مسى كنتــد و از كمتـرين تغييـرات برخوردار هستند.

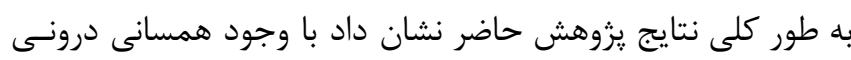
ضعيف ابعاد ابزار نكرش نسبت به يِيشخيرى از زخمه فشارى، با توجه

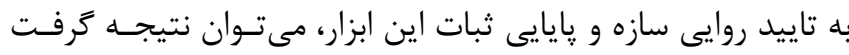

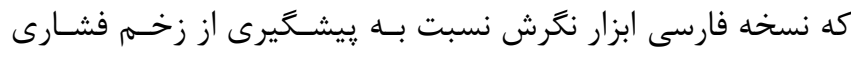

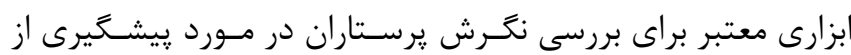

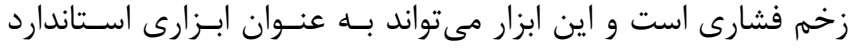

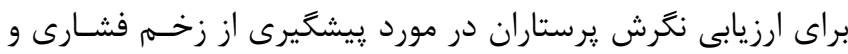
انجام ساير تحقيقات مرتبط با اين موضوع مورد استفاده قرار كيــرد.

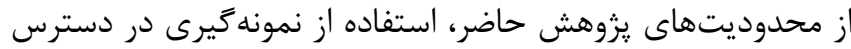

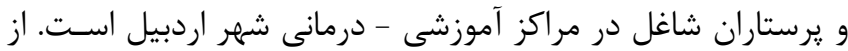

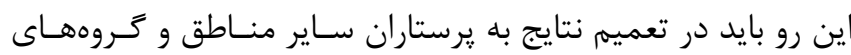

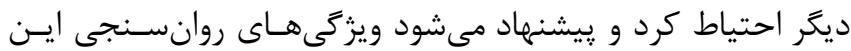
ابزار در ساير نقاط ايران و ساير گروهها نيز انجام گيرد.

\section{سهم نويسند}

مهدى حيدرزاده: راهنمايى در زمينه نغارش و ويرايش مقاله، نظارت بر تدوين مقاله و تصحيح آن، تجزيه و تحليل دادهها

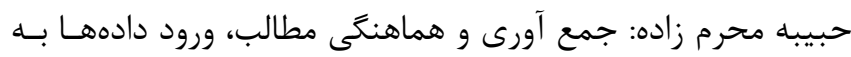
رايانه، تجزيه و تحليل دادهها، تهيه و تدوين نهايى مقاله نادر محرم زاده: جمع آورى دادهها

\section{تشكر و قدردانى}

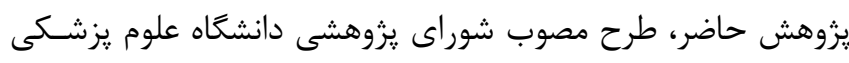

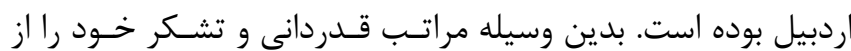

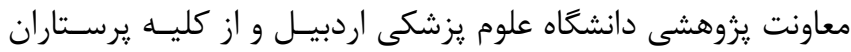

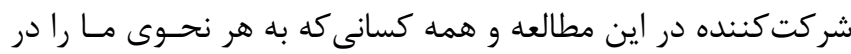
اجراى اين يزوهش يارى نمودند، اعلام مىنماييم.

\section{منابع}

1. WoundSource. National Pressure Ulcer Advisory Panel (NPUAP) Announces a Change in Terminology from Pressure Ulcer to Pressure Injury and Updates the Stages of Pressure Injury. April 21, 2016.

Available

at: http://www.woundsource.com/blog/national-pressureulcer-advisory-panel-npuap-announces-change-

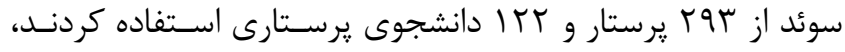

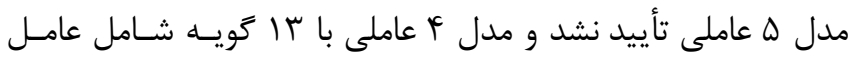

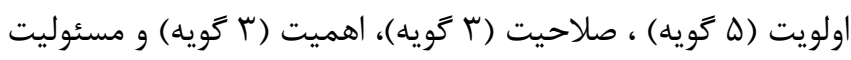

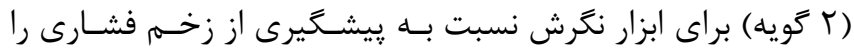

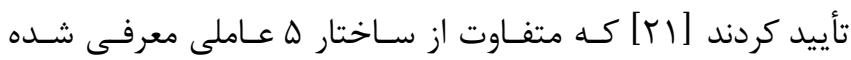

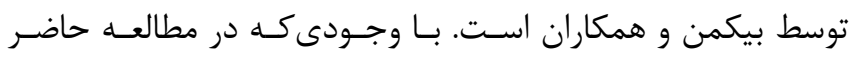

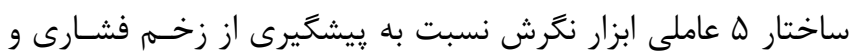

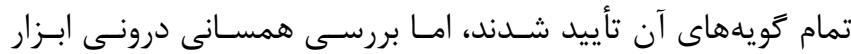

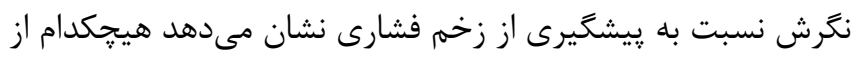

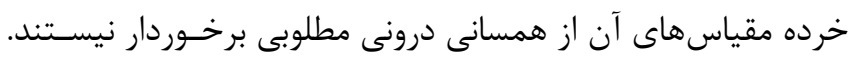

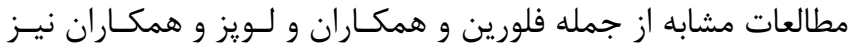

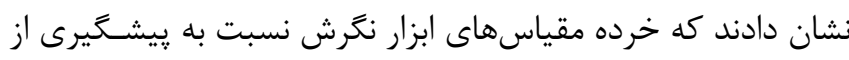

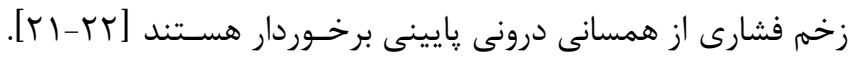

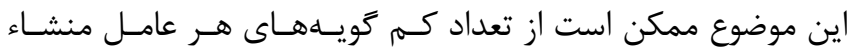

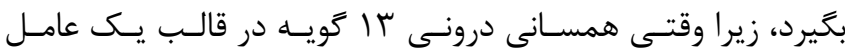

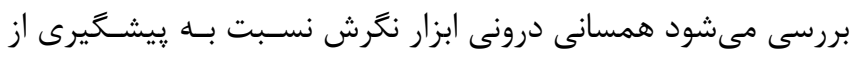

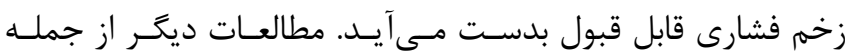
بيكمن و همكاران در بلزيك، لويز و همكـاران در اسـيانيا، كـريس و و

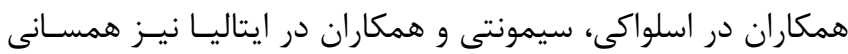

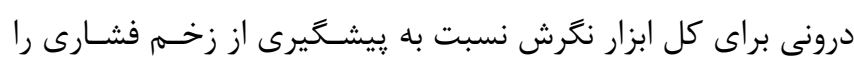

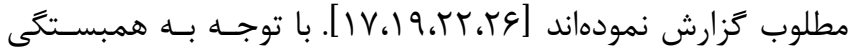

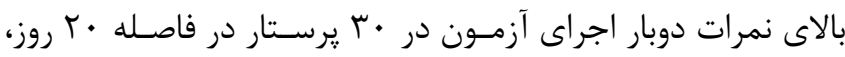

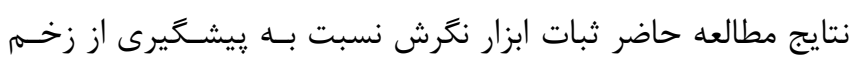

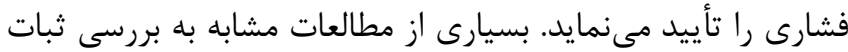
ابزار نكرش نسبت به يِيشخيرى از زخم فشارى اقدام نكـردهانـد، امـا

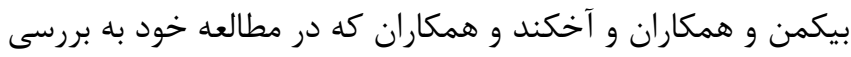

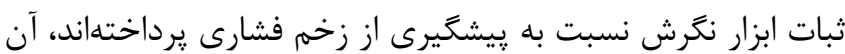

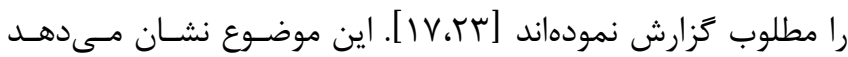

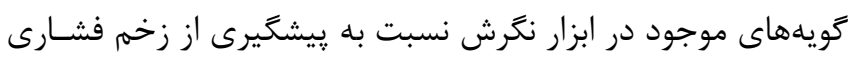

interminology- pressure-ulcer. [Last accessed December 31, 2020]

2. Jiang L, Li L, Lommel L. Nurses' knowledge, attitudes, and behaviors related to pressure injury prevention: A large scale cross sectional survey in mainland China. Journal of Clinical Nursing 2020;29:3311-24 
3. Han SH, Kim YS, Hwang J, Lee J, Song MR. Predictors of hospital- acquired pressure ulcers among older adult inpatients. Journal of Clinical Nursing 2018;27:3780-6

4. Karimian M, Sarokhani D, Sarokhani M, Sayehmiri K, Mortazavi Tabatabai SA. Prevalence of bedsore in Iran: a systematic review and metaanalysis. Journal of Mazandaran University of Medical Sciences 2016;26:202-10 [Persian]

5. Fox C. Living with a pressure ulcer: a descriptive study of patients' experiences. British Journal of Community Nursing 2002;7:10-22

6. Courvoisier DS, Righi L, Béné N, Rae AC, Chopard P. Variation in pressure ulcer prevalence and prevention in nursing homes: A multicenter study. Applied Nursing Research 2018;42:45-50

7. Tadiparthi S, Hartley A, Alzweri L, Mecci M, Siddiqui H. Improving outcomes following reconstruction of pressure sores in spinal injury patients: a multidisciplinary approach. Journal of Plastic, Reconstructive \& Aesthetic Surgery 2016;69:994-1002

8. Morehead D, Blain B. Driving hospitalacquired pressure ulcers to zero. Critical Care Nursing Clinics 2014;26:559-67

9. Li Z, Lin F, Thalib L, Chaboyer W. Global prevalence and incidence of pressure injuries in hospitalised adult patients: A systematic review and meta-analysis. International Journal of Nursing Studies 2020;105:103546

10. Amir Y, Lohrmann C, Halfens RJ, Schols JM. Pressure ulcers in four Indonesian hospitals: prevalence, patient characteristics, ulcer characteristics, prevention and treatment. International Wound Journal 2017;14:184-93

11. Samuriwo R, Dowding D. Nurses' pressure ulcer related judgements and decisions in clinical practice: a systematic review. International Journal of Nursing Studies 2014;51:1667-85

12. Hekmatpou D, Mehrabi F, Rahzani K, Aminiyan A. The effect of Aloe Vera gel on prevention of pressure ulcers in patients hospitalized in the orthopedic wards: a randomized triple-blind clinical trial. BMC Complementary and Alternative Medicine 2018;18:1-11

13. Köse I, Öztunç G. Knowledge of nurses working in intensive care units in relation to preventive interventions for pressure ulcer. International Journal of Caring Sciences 2016;9:677686
14. Tayyib NA, Coyer F, Lewis P. Pressure ulcers in the adult intensive care unit: a literature review of patient risk factors and risk assessment scales. Journal of Nursing Education and Practice 2013;3:28-42

15. Berlowitz D. Preventing pressure ulcers in hospitals: a tool kit for improving quality of care. Agency for Healthcare Research and Quality. 2012. Available at: http:// www.ahrq.gov/patient safety/settings/hospital/resource/

pressureulcer/tool/index.html. [Last accessed January 12, 2020]

16. Ajzen I. The theory of planned behavior. Organizational Behavior and Human Decision Processes 1991;50:179-211

17. Beeckman D, Defloor T, Demarré L, Van Hecke A, Vanderwee K. Pressure ulcers: development and psychometric evaluation of the attitude towards pressure ulcer prevention instrument (APuP). International Journal of Nursing Studies 2010;47:1432-41

18. Aslan A, van Giersbergen MY. Nurses' attitudes towards pressure ulcer prevention in Turkey. Journal of Tissue Viability 2016;25:66-73

19. Simonetti V, Comparcini D, Flacco ME, Di Giovanni P, Cicolini G. Nursing students' knowledge and attitude on pressure ulcer prevention evidencebased guidelines: a multicenter cross-sectional study. Nurse Education Today 2015;35:573-9

20. Üstün Y. Adapting the "attitude towards pressure ulcer prevention instrument" into Turkish and studying its validity and reliability [Master's Thesis]. Ege Üniversitesi Sa glık Bilimleri Fakültesi; 2013. [Turkish]

21. Florin $\mathrm{J}$, Bååth $\mathrm{C}$, Gunningberg L, Mårtensson G. Attitudes towards pressure ulcer prevention: a psychometric evaluation of the Swedish version of the APuP instrument. International Wound Journal 2016;13:655-62

22. López-Franco MD, Parra-Anguita L, CominoSanz IM, Pancorbo-Hidalgo PL. Attitudes of Spanish nurses towards pressure injury prevention and psychometric characteristics of the Spanish version of the APuP instrument. International Journal of Environmental Research and Public Health 2020; $17: 8543$

23. Akhkand SS, Seidi J, Ebadi A, Gheshlagh RG. Examination of the psychometric properties of the Persian version of the attitude towards pressure ulcer prevention instrument in nurses. Journal of Tissue Viability 2021;30:116-20 
24. Wild D, Grove A, Martin M, Eremenco S, McElroy S, Verjee-Lorenz A, et al. Principles of good practice for the translation and cultural adaptation process for patient-reported outcomes (PRO) measures: report of the ISPOR task force for translation and cultural adaptation. Value in Health 2005;8:94-104

25. Polit DF, Beck CT, Owen SV. Is the CVI an acceptable indicator of content validity? Appraisal and recommendations. Research in Nursing \& Health 2007;30:459-67

26. Grešš Halász B, Bérešová A, Tkáčová L, Magurová D, Lizáková L. Nurses' knowledge and attitudes towards prevention of pressure ulcers. International Journal of Environmental Research And Public Health 2021;18:1705-1714 